nitrogen in synthesis faster than it is supplied to the fruit, so the soluble nitrogen complement falls to a low level; thereafter it increases again to a new higher level at harvest. The composition of the soluble nitrogen fraction as stored at harvest is quite different from that which obtains in the inflorescence in which the fruit is being initiated. The latter emphasizes the amides (asparagine and glutamine) and histidine; the former emphasizes alanine and glutamine as the only prominent amide.

In Part 3, nitrogen compounds of the leaves are considered. The materials analysed were obtained from plants grown in sand cultures either supplied with full nutrient solution or with solutions deficient in each of the several major nutrient elements. The growth was continued long enough to establish the conditions characteristic of these nutrient treatments. Extracts of leaves, sampled according to their position on the axis, have becn made from plants grown in full nutrient solution; these extracts have been examined by chromatographic methods to detect and determine the various nitrogen compounds they contain.

The relative proportions of the soluble nitrogen compounds of the banana leaf are quite different from those of the fruit and, in response to the deficiency of specified mineral nutrients, both the total amount and the relative composition of the soluble nitrogen fraction are greatly affected. The results are interpreted in terms of the more active accumulation of soluble compounds in young leaves and the maintenance of a low amide (glutamine)-level in lcaves engaged in protein synthesis. The accumulation of soluble nitrogen compounds when growth and synthesis are arrested, and the relative accumulation of a speeified number of nitrogenous substances, due to the lack of a nutrient element, indicate that metabolic blocks in reaction pathways occur.

\title{
THE NUCLEUS OF THE ANDROMEDA NEBULA
}

TN 1959 the electronic camcra developed by Lalle1 mand and Duchesne in Paris was taken to the Lick Observatory on Mount Hamilton, California, and installed at the focus of the coude spectrograph of the 120-in. reflector. Among the objects chosen for observation was the nuclous of the Great Nebula in Andromeda, $M$ 31. The nucleus appeared on the slit as almost stellar, the inner parts of the nebula which aro normally so conspicuous on photographs being invisible.

The results of the observations have been reported by A. Lallemand, M. Duchesne and M. F. Walker (Pub. Astron. Soc. Pacific, 72, $76 ; 1960)$. The first spectrogram of the nucleus was made in vory good seeing when a stellar image was about half a second of arc in diameter, and the image of the nucleus was of diameter $4 \cdot 4^{\prime \prime}$. It showed at onco that the $H$ and $K$ lines of Ca II, which were the only ones clearly visible, were markedly inclined through the nucleus. Subsequent observations confirmed this. The observations have been interpreted on the assumption that the inclination of the lines is produced by the rotation of the nucleus, and, when converted to the equivalent velocities in circular orbits, the measures yield a maximum circular velocity of $87 \mathrm{~km}$. $/ \mathrm{sec}$. at a distance of $2 \cdot 2^{\prime \prime}$ from the centre of the nucleus.
Beyond this distance the velocity appears to decrease, a velocity of $42 \mathrm{~km}$./sec. being measured at $4 \cdot 2^{\prime \prime}$ from tho centre.

Previous observations of the rotation of $M 31$ refer to points farther from the centre, the innermost point being at $25^{\prime \prime}$ from the centre. The velocity apparently has a minimum somewhere in the region of $10^{\prime \prime}$ from the centre, a maximum at $3^{\prime}$, another minimum at $9^{\prime}$, and an increasing velocity for greater distances. If one assumes that the nucleus is spherical, of radius $2 \cdot 2^{\prime \prime}$, and that it rotates as a solid body with a maximum circular velocity of $87 \mathrm{~km}$./sec., its properties may be calculated. Its radius is $7 \cdot 4$ parsecs, its mass $1.3 \times 10^{7}$ solar masses, its average density $1.5 \times 10^{3}$ solar masses per cubic parsec and its rotation period $5.2 \times 10^{5}$ years. Its mass-toluminosity ratio is about $3 \cdot 6$ in solar units. The nucleus is therefore similar in size to a giant globular cluster, but one hundred times more massive and twenty times as dense and as luminous. It rotates one to two orders of magnitude faster than the rest of $M$ 31. The results indicate that the nucleus is a separate dynamical entity, although spectroscopic evidence suggests that its stellar composition is like that of the surrounding central regions.

\section{RESULTS OF THE OCCULTATION OF REGULUS BY VENUS, JULY 7, 1959}

\author{
By DR. G. de VAUCOULEURS and Dr. D. H. MENZEL \\ Harvard College Observatory, Cambridge, Mass.
}

\section{Ingress and Egress Observations}

$\mathrm{T}$ ABLE 1 gives the times $T_{1}, T_{2}$ of the halfintensity phase, $\Delta m=0.75$ mag., at ingress and egress derived from visual, photographic and photoelectric observations secured by members of the Harvard College Obsorvatory (stations $1 a, 2,3,4,5$,
7) and Smithsonian Astrophysical Observatory (stations $1 b, 6$ ) expeditions. Rain and clouds prevented observations at two other stations (Castel Gandolfo, Asiago). Additional observations (stations (8-24) wero reccived by letter or taken from published sources. Station co-ordinates, observers and sources are listed in Taklo 2. Published observations 